\title{
Oil removal from aqueous state by natural fibrous sorbent: an overview
}

\begin{abstract}
The threat of oil pollution increases with the expansion of oil exploration and production activities, as well as the industrial growth around the world. The study on the treatment of oily wastewater is a critical issue to the environmental protection as oil caused problems to the wastewater treatment facilities. Although oil particles can efficiently be removed by advanced technologies, the treatments are usually expensive and difficult to maintain. Adsorption and coalescence filtration are promising choice of treatment for its simplicity, effectiveness, and feasibility when appropriate sorbent is used. This review discusses the recent papers on the use of natural fibrous sorbent for removal of oil from wastewater, and its current development. With their excellent oil removal properties, environmental friendliness, easy availability, and feasibility, natural fibrous sorbents are an attractive alternative for oily wastewater treatment.
\end{abstract}

Keyword: Oily wastewater; Natural fibre; Adsorption; Coalescence 